\title{
Long non-coding RNA KCNQ10T1 mediates the growth of hepatocellular carcinoma by functioning as a competing endogenous RNA of miR-504
}

\author{
CHAO LI, RUNCHEN MIAO, JINGYAO ZHANG, KAI QU and CHANG LIU \\ Department of Hepatobiliary Surgery, First Affiliated Hospital of Xi'an Jiaotong University, \\ Xi'an, Shaanxi 710061, P.R. China
}

Received December 1, 2017; Accepted February 23, 2018

DOI: $10.3892 /$ ijo.2018.4313

\begin{abstract}
Accumulating evidence highlights the fact that long non-coding RNAs (lncRNAs) serve as critical factors in the growth of hepatocellular carcinoma (HCC). The dysregulation of 1ncRNA KCNQ1 opposite strand/antisense transcript 1 (KCNQ1OT1) has been reported in numerous human benign diseases. However, the role of KCNQ1OT1 in human HCC remains poorly understood. In this study, we demonstrated that the expression of KCNQ1OT1 was abnormally increased in HCC tissues. The ectopic high expression of KCNQ1OT1 was associated with liver cirrhosis, a larger tumor size, an advanced TNM stage, and a worse overall survival and tumor-free survival. For the first time, to the best of our knowledge, we report that KCNQ1OT1 knockdown results in a decreased cell viability and colony formation ability, and an increased rate of apoptosis in vitro. The results from our in vivo results demonstrated that KCNQ1OT1 silencing attenuated tumor growth by impairing cell proliferation. Additionally, we found that KCNQ1OT1 exerted its effects partly by relying on the microRNA-504 (miR-504)-mediated regulation of cyclin-dependent kinase 16 (CDK16), in addition to the regulation of the glycogen synthase kinase $3 \beta$ $(\mathrm{GSK} 3 \beta) / \beta$-catenin/Bcl-2 signaling pathway. The present study revealed the functions and mechanisms of action of lncRNA KCNQ1OT1 regarding its role in promoting the growth of HCC. Thus, lncRNA KCNQ1OT1 may prove to be a potential therapeutic target for human HCC.
\end{abstract}

\section{Introduction}

Hepatocellular carcinoma (HCC) remains a serious health burden worldwide (1). In China, $>466,100$ new cases and

Correspondence to: Professor Chang Liu, Department of Hepatobiliary Surgery, First Affiliated Hospital of Xi'an Jiaotong University, 277 Yanta West Road, Xi'an, Shaanxi 710061, P.R. China E-mail: liuchangdoctor@163.com

Key words: IncRNA KCNQ1OT1, hepatocellular carcinoma, growth, microRNA-504, CDK16
422,100 HCC-associated deaths were reported in 2015 (2). Clinical experience indicates that patients affected by tumors of a large size usually have a poor prognosis (3). This may be ascribed to two main reasons: The loss of surgical opportunity due to the large tumor size, and fatal complications following a massive hepatectomy. Thus, the control of tumor growth is a critical strategy for improving the prognosis of patients with HCC.

Long non-coding RNAs (lncRNAs) are a class of abundant and largely uncharacterized non-protein-coding RNAs that are $>200$ nucleotides in length (4). IncRNAs have been demonstrated to regulate cell proliferation in HCC (5). lncRNA TUG1 has been shown to promote HCC growth in vitro and in vivo by epigenetically suppressing Kruppel-like factor 2 (KLF2) transcription (6). IncRNA HOTAIR has been shown to increase cell viability by enhancing glucose transporter isoform 1 (GLUT1)-mediated glycolysis (7). lncRNA KCNQ1 opposite strand/antisense transcript 1 (known as KCNQ1OT1) was initially identified as an aberrantly expressed lncRNA in Beckwith-Wiedemann syndrome, which is an overgrowth disorder usually presenting in the embryonic stages (8). Recently, KCNQ1OT1 was found to be required for the proliferation of cancer cells. In breast cancer, a high KCNQ1OT1 expression has been shown to be associated with a large tumor size (9). Yoshizawa et al (10) found that pyrrole-imidazole polyamide bound to the CCAAT boxes of the KCNQ1OT1 promoter region to silence its expression. Moreover, KCNQ1OT1 silencing by this drug induced the death of Wilms' tumor-derived G401 cells (10). These results indicate that KCNQ1OT1 may be a novel therapeutic target for patients with high intra-tumoral levels of KCNQ1OT1.

In the present study, we confirmed the cell growth-related lncRNA KCNQ1OT1 to be critical for HCC cell growth in vitro and in vivo. The overexpression of KCNQ1OT1 was found to be associated with a large tumor size and a poor prognosis of patients with HCC. Additionally, KCNQ1OT1 was found to function as a competing endogenous RNA sponge for microRNA-504 (miR-504). Thus, cyclin-dependent kinase 16 (CDK16), a downstream target of miR-504, was harbored by KCNQ1OT1 to facilitate its tumor growth-promoting effects. 


\section{Materials and methods}

Clinical samples and cell lines. A total of 50 pairs of liquid nitrogen-stored HCC tissues and matched tumor-adjacent tissues were collected from 30 male patients and 20 female patients at the Department of Hepatobiliary Surgery, First Affiliated Hospital of Xi'an Jiaotong University (Xi'an, China) between January 2011 and January 2013. Written informed consent was obtained from all patients enrolled in this study. The use of clinical samples was approved by the Ethics Committee of First Affiliated Hospital of Xi'an Jiaotong University. In total, 5 HCC cell lines (Huh-7, SMMC-7721, Hep3B, MHCC97-H and MHCC97-L), the human immortalized normal hepatocyte cell line, LO2, and 293T cells were obtained from the Shanghai Institute of Biochemistry and Cell Biology, Chinese Academy of Sciences (Shanghai, China), and maintained in our laboratory. The cells were cultured in Dulbecco's modified Eagle's medium (DMEM) containing $10 \%$ fetal bovine serum (FBS) (both from Gibco, Carlsbad, CA, USA) and 1\% V/V penicillin/streptomycin (MilliporeSigma, Bedford, MA, USA) in a humidified atmosphere containing $5 \% \mathrm{CO}_{2}$ at $37^{\circ} \mathrm{C}$.

Cancer Genome Atlas dataset. Gene Expression Omnibus (GEO) data for 95 HCC samples and 39 normal liver samples were collected from the dataset entitled Gene Expression Profiles of Human Hepatocellular Carcinoma (GSE45436), and pre-analyzed using R2: Genomics Analysis and Visualization Platform (http://r2.amc.nl).

Reverse transcription-quantitative PCR (RT-qPCR). Total RNA from tissues and cells was isolated using the miRNeasy Mini kit (Qiagen, Germantown, MD, USA). The KCNQ1OT1 levels, miR-504 levels and CDK16 mRNA levels were measured by RT-PqCR using a SuperScript III Reverse Transcriptase kit (Invitrogen, Carlsbad, CA, USA) and an iTaq Universal SYBR-Green Supermix kit (Bio-Rad Laboratories, Hercules, CA, USA). The Bulge-Loop miR-504 qPCR Primer Set was purchased from RiboBio Co., Ltd. (Guangzhou, China). Other primer sequences were as follows: KCNQ1OT1 forward, 5'-CTTTGCAGCAACCTCCTTGT-3' and reverse, 5'-TGG GGTGAGGGATCTGAA-3'; CDK16 forward, 5'-CCGTCGT GTCAGCCTATCT-3' and reverse, 5'-CTTCTCCGTGTG GATAATGTCA-3'; and GAPDH forward, 5'-CCAGGGCTG CTTTTAACTCT-3' and reverse, 5'-GGACTCCACGACGTA CTCA-3'. An ABI 7500 Real-Time PCR System (Applied Biosystems, Foster City, CA, USA) was used to perform the qPCR assay. The thermocycling conditions were as follows: A holding stage at $50^{\circ} \mathrm{C}$ for $2 \mathrm{~min}$, and $95^{\circ} \mathrm{C}$ for $10 \mathrm{~min}$; PCR stage (40 cycles) at $95^{\circ} \mathrm{C}$ for $15 \mathrm{sec}$, and $60^{\circ} \mathrm{C}$ for $1 \mathrm{~min}$. The $2^{-\Delta \Delta C q}$ method (11) was used to calculate the relative gene expression normalized by GAPDH.

Cell transduction and transfection. KCNQ1OT1-specific shRNA (sh-KCNQ1OT1) and negative control shRNA (sh-Ctrl) were designed and synthesized by GeneCopoeia (Guangzhou, China), and cloned into the psi-LVRH1GP lentiviral vectors. Recombinant lentiviruses were transduced into the Huh-7 and SMMC-7721 cells. miR-504 mimics (miR-504), negative control mimics (miR-Ctrl), miR-504 inhibitor
(anti-miR-504) and a negative control inhibitor (anti-miR-Ctrl) were purchased from RiboBio Co., Ltd. The vectors, mimics and respective inhibitors were transfected into the cells using Lipofectamine 3000 reagent (Invitrogen/Thermo Fisher Scientific, Inc., Waltham, MA, USA), as described in a previous study of ours (12).

Cell counting kit-8 (CCK-8) assay. The cancer cells were seeded into a 96-well plate at 2,000/well in quintuplicate, and cell viability was determined by CCK-8 assay (E606335; Sangon, Shanghai, China) at 0,24, 48 and $72 \mathrm{~h}$. The absorbance of the samples was measured using a model 550 microplate reader (Bio-Rad Laboratories) at a wavelength of $450 \mathrm{~nm}$.

Colony formation assay. A total of 400 cells were seeded into a 6-well plate and maintained in DMEM containing 10\% FBS for 2 weeks. Cell colonies were fixed with $20 \%$ methanol and stained with $0.1 \%$ crystal violet at room temperature for $15 \mathrm{~min}$. The colonies were counted using a ELIspot Bioreader 5000 (BIO-SYS, Karben, Germany).

Flow cytometry. Apoptotic cells were stained at the time point of $72 \mathrm{~h}$ of transfection with the Annexin V-PE/7-AAD apoptosis detection kit (KGA-1017; KeyGEN, Nangjing, China). The cells $\left(2 \times 10^{5}\right)$ were suspended with $50 \mu \mathrm{l}$ binding buffer, and received the following treatments: $5 \mu 1 /$ sample 7-AAD with 15 min incubation; $1 \mu \mathrm{l} / \mathrm{sample}$ Annexin V-PE and $450 \mu \mathrm{l} / \mathrm{sample}$ binding buffer with $15 \mathrm{~min}$ of incubation. Positively stained cells were detected using a BD FACSCanto II Flow Cytometer (BD Biosciences, Franklin Lakes, NJ, USA).

Apo-ONE ${ }^{\circledR}$ homogeneous caspase-3/7 assay. A total of $1 \times 10^{4}$ cells $(100 \mu \mathrm{l})$ were seeded into a 96-well plate. This was followed by the thawing and mixing of the Caspase Substrate and Apo-ONE ${ }^{\circledR}$ Caspase-3/7 Buffer to produce the Apo-ONE ${ }^{\circledR}$ Caspase-3/7 Reagent according to the manufacturer's instructions (Promega, Madison, WI, USA) and then by the addition of $100 \mu \mathrm{l}$ reagent to each well. The wells were then incubated at room temperature for $4 \mathrm{~h}$. The fluorescence of each well at $499 \mathrm{~nm}$ was measured using a model 550 microplate reader (Bio-Rad Laboratories).

In vivo tumor growth assays. The in vivo experiments were performed after obtaining ethics approval from the Biomedical Ethics Committee of Xi'an Jiaotong University Health Science Center. A total of $12 \mathrm{BALB} / \mathrm{cA}$ male nude mice (6 weeks old, weighing 16-18 g) were obtained from the Laboratory Animal Center of Xi'an Jiaotong University Health Science Center, kept in sterilized cages in an appropriate environment $\left(25^{\circ} \mathrm{C}, 45 \%\right.$ humidity, 12:12 light:dark cycles), and fed a regular chow diet with water available ad libitum. The mice were randomly divided into 4 groups as follows: The Huh-7 sh-control group, Huh-7 sh-KCNQ1OT1 group, SMMC-7721 sh-control group and the SMMC-7721 sh-KCNQ1OT1 group. Modified Huh-7 and SMMC-7721 cells were subcutaneously injected into the outside of the right hind limb of the nude mice to establish tumor xenograft models. The tumor diameter was measured using a vernier caliper every 7 days to monitor the tumor growth from the time of the injection. The tumor volume was calculated as follows: (length $\mathrm{x}$ width $\left.{ }^{2}\right) / 2$. All 
mice were kept under the aforementioned conditions. After 4 weeks, the mice were sacrificed by $\mathrm{CO}_{2}$ euthanasia (the flow rate of $\mathrm{CO}_{2}$ was $20 \%$ displacement $/ \mathrm{min}$ ). The final weights of the mice at the time of sacrifice were 20-23 g. Each mouse only had a single tumor nodule. The maximum diameter of a single tumor measured was $14.9 \mathrm{~mm}$, and the largest single tumor volume was $1,251.31 \mathrm{~mm}^{3}$. Paraformaldehyde-fixed paraffin-embedded mouse subcutaneous tumor tissue sections were created as previously described (12).

Immunohistochemistry. Specific Ki-67 rabbit anti-human primary antibodies (\#9027) were purchased from Cell Signaling Technology (Danvers, MA, USA) and diluted 1:100 with PBS to label the antigens at $4^{\circ} \mathrm{C}$ overnight. Biotinylated goat anti-rabbit secondary antibodies were used to label the combined primary antibodies. Complexes were detected using HRP-streptavidin conjugates and visualized with DAB. In total, 20 high power (x400) fields were randomly selected to calculate the total cell numbers and $\mathrm{Ki}$-67-positive cell numbers. The percentage of $\mathrm{Ki}-67$-positive cells was identified as the Ki-67 index.

Western blot analysis. The cells were lysed with RIPA buffer (HEART Biotech, Xi'an, China) and quantified using a BCA protein assay kit II (\#5000002; Bio-Rad Laboratories). Protein samples $(40 \mu \mathrm{g})$ were separated by $10 \%$ SDS-PAGE and transferred onto nitrocellulose membranes (Invitrogen/Thermo Fisher Scientific, Inc.) using a Bio-Rad tank blotting system (Bio-Rad Laboratories). The membranes were incubated with the appropriate primary antibodies at a 1:1,000 dilution overnight at $4^{\circ} \mathrm{C}$. Horseradish peroxidase-conjugated secondary antibodies at a 1:2,000 dilution were used to incubate the membranes for $1 \mathrm{~h}$ at room temperature, after washing them with TBST 3 times for $10 \mathrm{~min}$. The targeting proteins on the membrane were visualized with ECL reagents (Millipore, Plano, TX, USA). The primary antibodies used in this study were purchased from Cell Signaling Technology, and included those against glycogen synthase kinase $3 \beta$ (GSK3 $\beta$ ) (\#5676), GSK3ßser9 (\#9322), $\beta$-catenin (\#8480), Bcl-2 (\#2872) and GAPDH (\#5174). The secondary antibodies used, included the Amersham ECL Donkey Anti-Mouse IgG Horseradish Peroxidase-Linked Species-Specific Whole Antibody (NA931; GE Healthcare Life Sciences, Pittsburgh, PA, USA). The relative band density was determined using ImageJ software (NIH, Bethesda, ML, USA).

Luciferase reporter assay. StarBase v2.0 (http://starbase. sysu.edu.cn/mirLncRNA.php) software was used to predict the target mRNA of miR-504. The wild-type (Wt) fragments from KCNQ1OT1 containing the predicted miR-504 binding sites and the corresponding mutant-type (Mt) fragments were cloned into the pEZX-MT06 vectors (RiboBio Co., Ltd.), respectively. Similarly, three online MicroRNA targets prediction tools, TargetScan (http://www.targetscan.org/), PicTar (http://www.pictar.org/) and MiRanda (http://www. microrna.org/), were used to located the binding sites between miR-1271 and PTP4A1 3'-UTR region. The Wt fragment from the CDK16 3'-UTR region and the corresponding Mt fragment were cloned into the pEZX-MT06 vectors. The vectors and miR-504 mimics were then co-transfected into $293 \mathrm{~T}$ cells using
Lipofectamine 3000 reagent, and the Luc-Pair Duo-Luciferase assay kit 2.0 (GeneCopoeia) was used to determine the relative Rluc/Luc ratio.

Statistical analysis. Continuous data are presented as the means \pm SD. Statistical analysis was performed using SPSS version 21.0 (SPSS Inc., Chicago, IL, USA) and GraphPad Prism 5 (GraphPad Software, Inc., La Jolla, CA, USA). Correlations between KCNQ1OT1 and clinical characteristics were analyzed using the Pearson's Chi-squared test. The differences between 2 groups were analyzed using a Student's t-test. ANOVA was used to compare the data from multiple groups. The Bonferroni method was used as the post hoc test. Survival analysis was performed using a Kaplan-Meier curve. The hazard ratio and confidence interval were determined using the log-rank (Mantel-Cox) test. The respective correlations between KCNQ1OT1 and miR-504, and CDK16 mRNA levels were analyzed by Spearman's correlation analysis. A value of $\mathrm{P}<0.05$ was considered to indicate a statistically significant difference.

\section{Results}

Expression of KCNQ1OT1 is increased in HCC tissues and predicts a worse prognosis. The expression of KCNQ1OT1 in human tissues was screened using RT-qPCR. We found that KCNQ1OT1 expression was significantly increased in the HCC tissues compared with in the adjacent non-tumor tissues (Fig. 1A, P<0.001). To confirm the results obtained by our PCR analysis, we further analyzed KCNQ1OT1 expression data available in the GSE dataset (GSE45436). As expected, KCNQ1OT1 expression was also upregulated in those $95 \mathrm{HCC}$ tissues (Fig. 1B, $\mathrm{P}=0.002$ ). Additionally, in comparison to the HCC tissues obtained from small tumors ( $<5 \mathrm{~cm}$ in diameter), the KCNQ1OT1 levels were significantly higher in the tissues obtained from large tumors ( $\geq 5 \mathrm{~cm}$ in diameter) (Fig. 1C, $\mathrm{P}<0.001)$. This result indicated that KCNQ1OT1 may be associated with tumor growth in HCC.

Therefore, in order to further determine the clinical and prognostic significance of KNQ1OT1 in HCC, we selected the mean value of KCNQ1OT1 (2.112) as a cut-off value to divide the 50 patients with HCC into a low KCNQ1OT1 expression group ( $\mathrm{n}=22,<2.112)$ and a high KCNQ1OT1 expression group $(\mathrm{n}=28, \geq 2.112)$. The correlations between KCNQ1OT1 expression and the clinical characteristics were analyzed using the Chi-squared test (Table I). High expression of KCNQ1OT1 was confirmed to be associated with liver cirrhosis $(\mathrm{P}=0.027)$, a large tumor size $(>5 \mathrm{~cm}, \mathrm{P}<0.001)$ and an advanced TNM stage (stages III and IV, $\mathrm{P}=0.002$ ). Moreover, patients with HCC and a high KCNQ1OT1 expression experienced a shorter overall survival (Fig. 1D; hazard ratio, 2.962; 95\% confidence interval, 1.440-6.091; $\mathrm{P}=0.003$ ) and tumor-free survival (Fig. 1E; hazard ratio, 2.588; 95\% confidence interval, 1.282-5.221; $\mathrm{P}=0.008)$ than patients with a low KCNQ1OT1 expression. These findings thus suggest that KCNQ1OT1 has the potential to predict the outcomes of patients with HCC.

KCNQ1OT1 enhances HCC proliferation and tumorigenicity in vitro and in vivo. Next, in vitro cell proliferation and apoptosis experiments were carried out to determine 


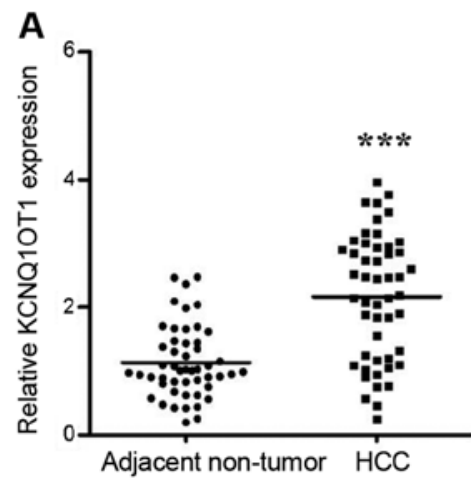

D
B

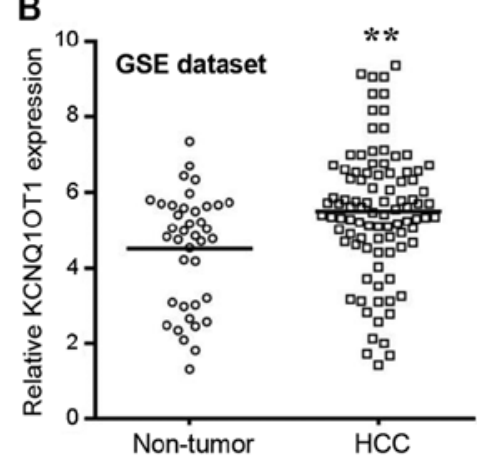

C

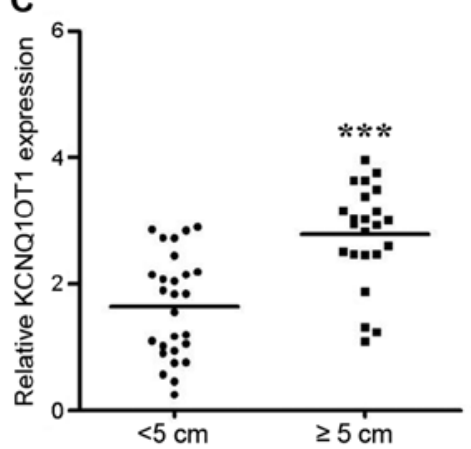

E
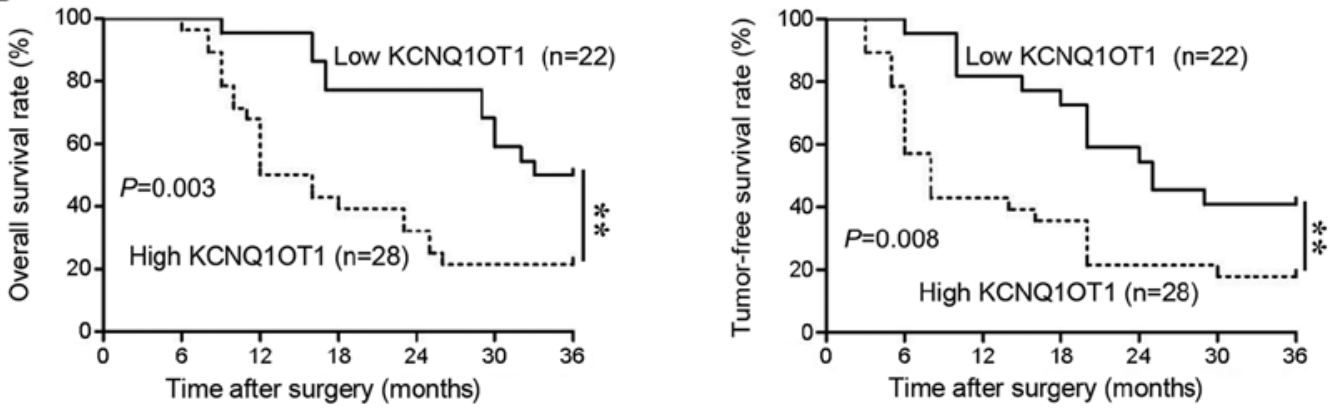

Figure 1. Expression and Kaplan-Meier analysis of KCNQ1OT1 in hepatocellular carcinoma (HCC). (A) The expression of KCNQ1OT1 in 50 paired HCC tissues and tumor-adjacent tumor tissues. (B) Upregulation of KCNQ1OT1 was detected in 95 HCC samples from the GSE45436 dataset. (C) The average expression level of KCNQ1OT1 was higher in the larger-sized ( $\geq 5 \mathrm{~cm}$ in diameter) HCC tumors. (D and E) Correlations between KCNQ1OT1 expression and (D) overall survival or (E) tumor-free survival were analyzed using Kaplan-Meier survival curves. ${ }^{* *} \mathrm{P}<0.01$ and ${ }^{* * *} \mathrm{P}<0.001$.

the role of KCNQ1OT1 in tumor growth. We first detected KCNQ1OT1 expression in LO2 cells and in 5 HCC cell lines, and found that KCNQ1OT1 expression was upregulated in all the HCC cell lines, apart from the Hep3B and MHCC97-L cell lines (Fig. 2A, P<0.05, respectively). Two HCC cell lines with high KCNQ1OT1 expression levels, namely Huh-7 and SMMC-7721, were transduced with a KCNQ1OT1-specific shRNA lentivirus. The PCR results revealed that KCNQ1OT1 expression was silenced in these two cell lines following transduction (Fig. 2B, $\mathrm{P}<0.01$, respectively).

KCNQ1OT1 knockdown significantly suppressed the viability of the Huh-7 and SMMC-7721 cells (Fig. 2C, P<0.05, respectively). The capacity of the Huh-7 and SMMC-7721 cells for clone formation was also inhibited by KCNQ1OT1 knockdown (Fig. 2D, $\mathrm{P}<0.05$, respectively). The effect of KCNQ1OT1 on cell apoptosis was also examined in this study. We found that KCNQ1OT1 silencing increased the percentage of apoptotic cells (Fig. 2E, $\mathrm{P}<0.05$, respectively), as well as caspase-3/7 activity (Fig. $2 \mathrm{~F}, \mathrm{P}<0.05$, respectively) in the Huh-7 and SMMC-7721 cells.

The cells in which KCNQ1OT1 was stably knocked down were implanted into nude mice to examine the growth of HCC cells in vivo. Compared with the mice in the control group, the tumors in mice injected with KCNQ1OT1 shRNA-transduced Huh-7 (Fig. 3A, upper panel) and SMMC-7721 (Fig. 3A, lower panel) cells grew at a slower rate during the 4-week observation period $(\mathrm{P}<0.05$, respectively). Subsequently, a classical proliferation marker, Ki-67, was stained in order to measure the cell proliferation in the tumors xenografts. As was expected, the Ki-67-positive percentage of Huh-7 (Fig. 3B, upper panel) and SMMC-7721 (Fig. 3B, lower panel) cells in the tumor tissues derived from cells in which KCNQ1OT1 was knocked down was significantly lower than that in the tumor tissues derived from the negative controls $(\mathrm{P}<0.05$, respectively). These results thus suggest that KCNQ1OT1 promotes HCC growth by promoting cell proliferation and inhibiting apoptosis.

KCNQ1OT1 drives tumor growth in HCC by sponging $m i R-504$. IncRNAs can bind to miRNAs and function as sponges to regulate the expression of certain miRNAs. As shown in Fig. 4A, we identified that the seed region of miR-504 (2-8 bp from 5') has putative binding sites for KCNQ1OT1. We cloned these sequences into reporter vectors; the reporter vector and miR-504 were co-transfected into $293 \mathrm{~T}$ cells. The luciferase activities were significantly inhibited by the miR-504 mimics $(\mathrm{P}<0.05$, respectively). The expression of miR-504 was upregulated when KCNQ1OT1 was knocked down in the Huh-7 and SMMC-7721 cells (Fig. 4B, P<0.05, respectively). We also found that the overexpression of miR-504 decreased KCNQ1OT1 expression, whereas miR-504 knockdown increased KCNQ1OT1 expression (Fig. 4C, $\mathrm{P}<0.05$, respectively). Furthermore, a significant inverse correlation was demonstrated between the KCNQ1OT1 and miR-504 levels (Fig. 4D, $r=-0.337$; 95\% confidence interval, -0.5680 to $-0.05574 ; \mathrm{P}=0.017$ ). In addition, the miR-504 inhibitor was transfected into the Huh-7 and SMMC-7721 cells in which KCNQ1OT1 was knocked down to further examine whether KCNQ1OT1-induced HCC cell proliferation could be ascribed to miR-504 inhibition. Even though KCNQ1OT1 knockdown inhibited the viability and promoted the apoptosis of Huh-7 and SMMC-7721 cells, these effects were markedly abrogated by miR-504 inhibition (Fig. 4E and F, P $<0.05$, respectively). 

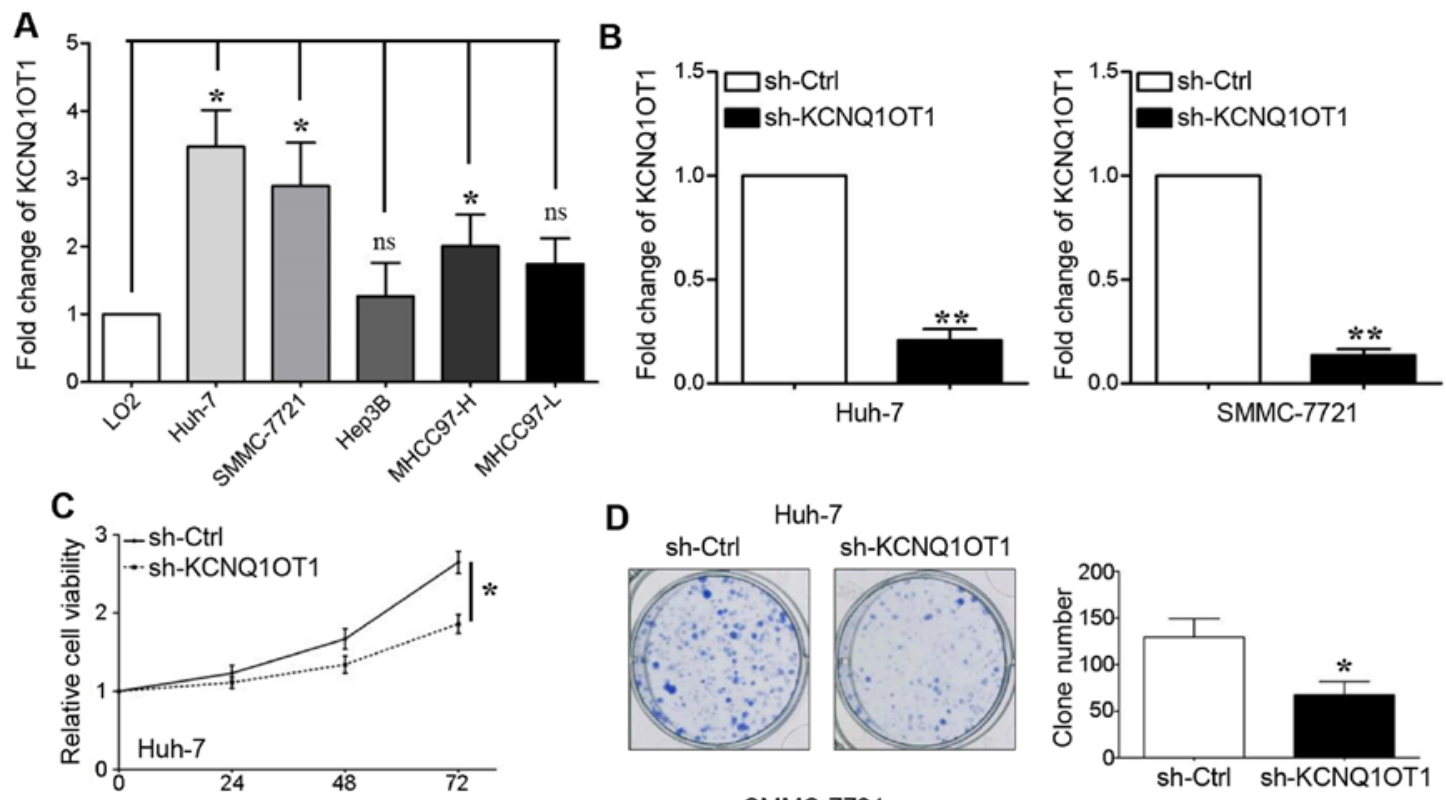

D
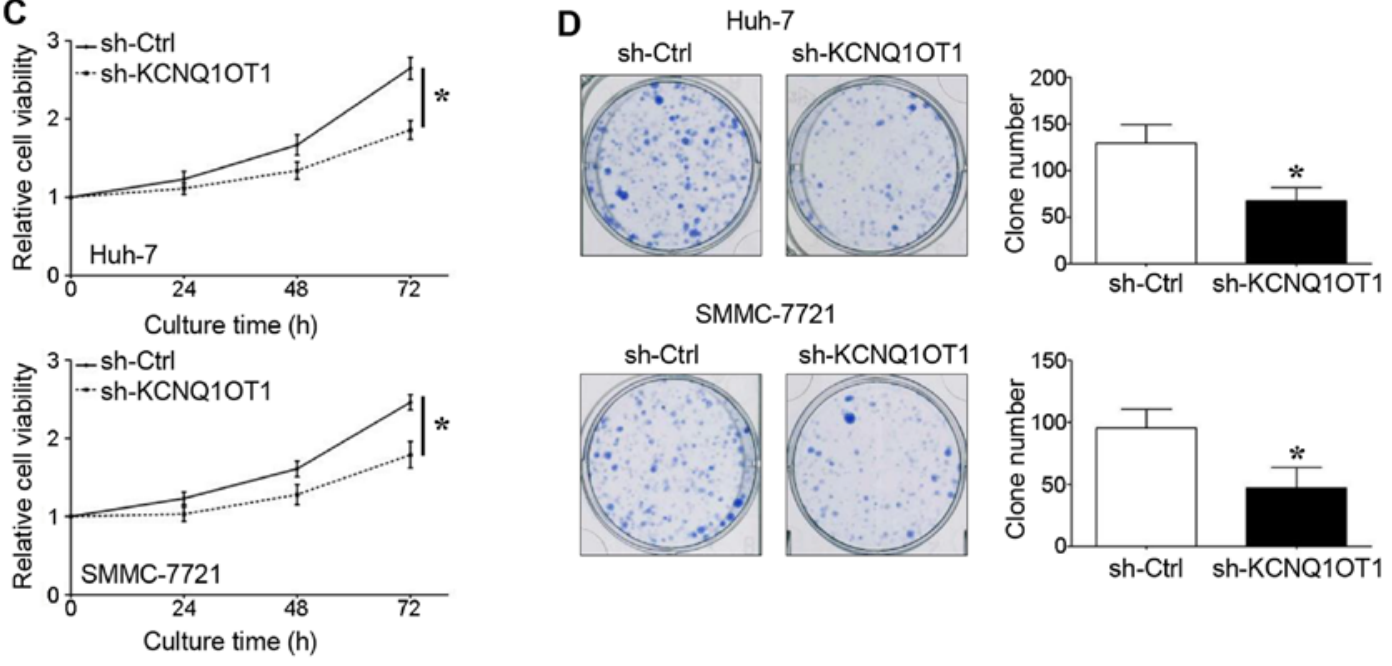

SMMC-7721
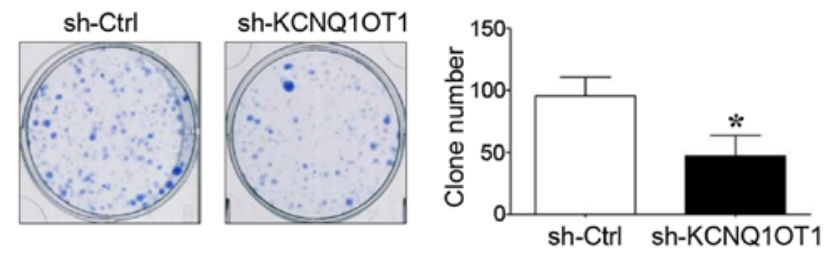

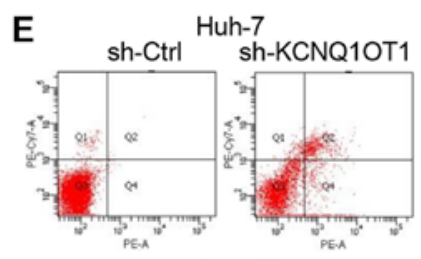

SMMC-7721

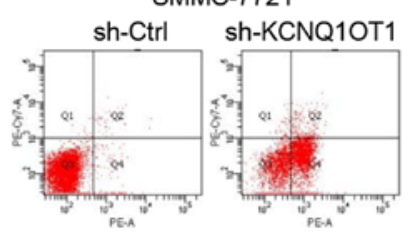

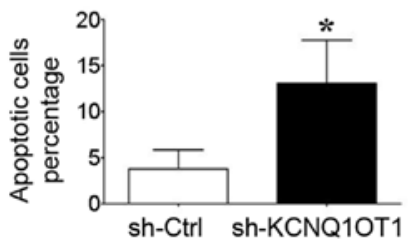

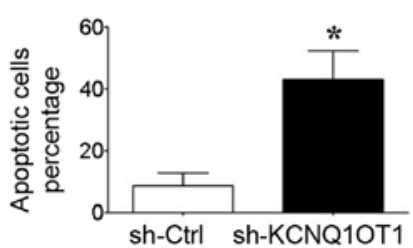

$\mathbf{F}$

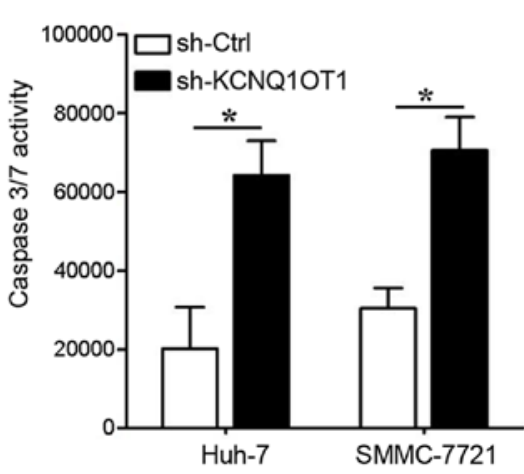

Figure 2. Downregulation of KCNQ1OT1 inhibits cell proliferation and promotes apoptosis in vitro. (A) KCNQ1OT1 expression was measured in normal liver cells (LO2) and HCC cell lines (Huh-7, SMMC-7721, Hep3B, MHCC97-H and MHCC97-L). (B) KCNQ1OT1 expression was downregulated by shRNA in Huh-7 and SMMC-7721 cells. (C) The viabilities of Huh-7 and SMMC-7721 cells transfected with KCNQ1OT1 shRNA were determined by CCK-8 assay. (D) KCNQ1OT1 knockdown regulated the colony formation ability of the Huh-7 and SMMC-7721 cells. (E) The apoptotic percentage of Huh-7 and SMMC-7721 cells following KCNQ1OT1 knockdown was measured by flow cytometric analysis. (F) KCNQ1OT1 knockdown affected caspase-3/7 activity in the Huh-7 and SMMC-7721 cells. ${ }^{*} \mathrm{P}<0.05$ and ${ }^{* *} \mathrm{P}<0.01$; ns, no statistical significance.

CDK16 is a direct target of $\mathrm{miR}-504$ in HCC. To explore the underlying mechanisms of the KCNQ1OT1/miR-504 axis in tumor growth regulation, we used the TargetScan (http://www. targetscan.org/), PicTar (http://www.pictar.org/) and MiRanda (http://www.microrna.org/) algorithms to predict the putative targets and binding sites of miR-504. In silico analyses revealed that the CDK16 3'-UTR contains a corresponding sequence to the miR-504 seed region (Fig. 5A). Indeed, relative luciferase activity was significantly inhibited only when we co-transfected the wild-type CDK16 3'-UTR reporter vector with miR-504 mimics (Fig. 5B, 2nd bar from the left; $\mathrm{P}<0.05$ ) or KCNQ1OT1 shRNA (Fig. 5B, 3rd bar from the left; $\mathrm{P}<0.05$ ).
Moreover, both miR-504 overexpression and KCNQ1OT1 knockdown markedly inhibited CDK16 mRNA (Fig. 5C, 2nd and 4th bars from the left; $\mathrm{P}<0.05$, respectively) and protein (Fig. 5D, lanes 2 and 4; $\mathrm{P}<0.05$, respectively) expression. To decrease CDK16 expression, miR-504 overexpression and KCNQ1OT1 knockdown functioned synergistically (Fig. 5C, 5th bar from the left; and Fig. 5D, lane 5; $\mathrm{P}<0.05$, respectively). By contrast, the inhibition of miR-504 expression increased CDK16 mRNA (Fig. 5C, 3rd bar from the left; $\mathrm{P}<0.05$ ) and protein (Fig. 5D lane 3; $\mathrm{P}<0.05$ ) expression, and abrogated the effects of KCNQ1OT1 knockdown on CDK16 expression (Fig. 5C, 6th bar from the left; and Fig. 5D, lane 6). A 
A
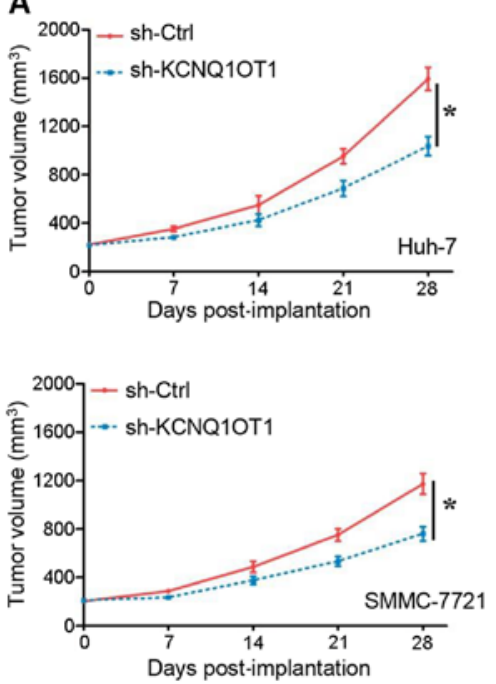

B

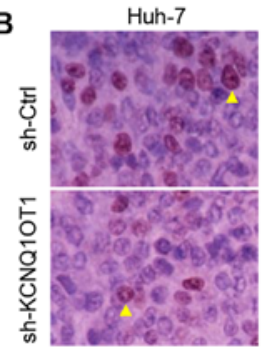

SMMC-7721

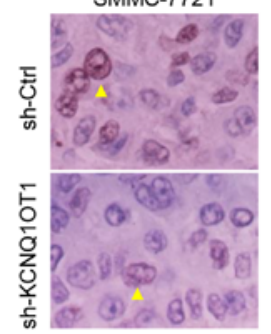

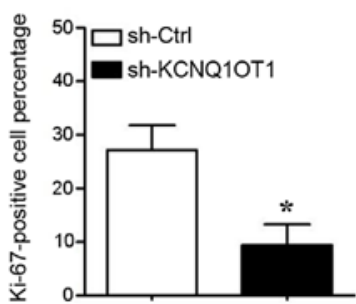

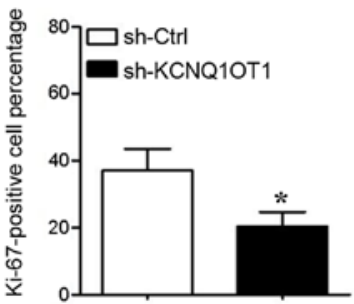

Figure 3. KCNQ1OT1 knockdown inhibits tumor growth in vivo. (A) Downregulation of KCNQ1OT1 affected the volumes of the subcutaneous tumors formed from the Huh-7 and SMMC-7721 cells. (B) Subcutaneous tumor sections were immunohistochemically stained for Ki-67 to measure cell proliferation. Ki-67-positive cells are labeled with yellow arrows. ${ }^{*} \mathrm{P}<0.05$.

A
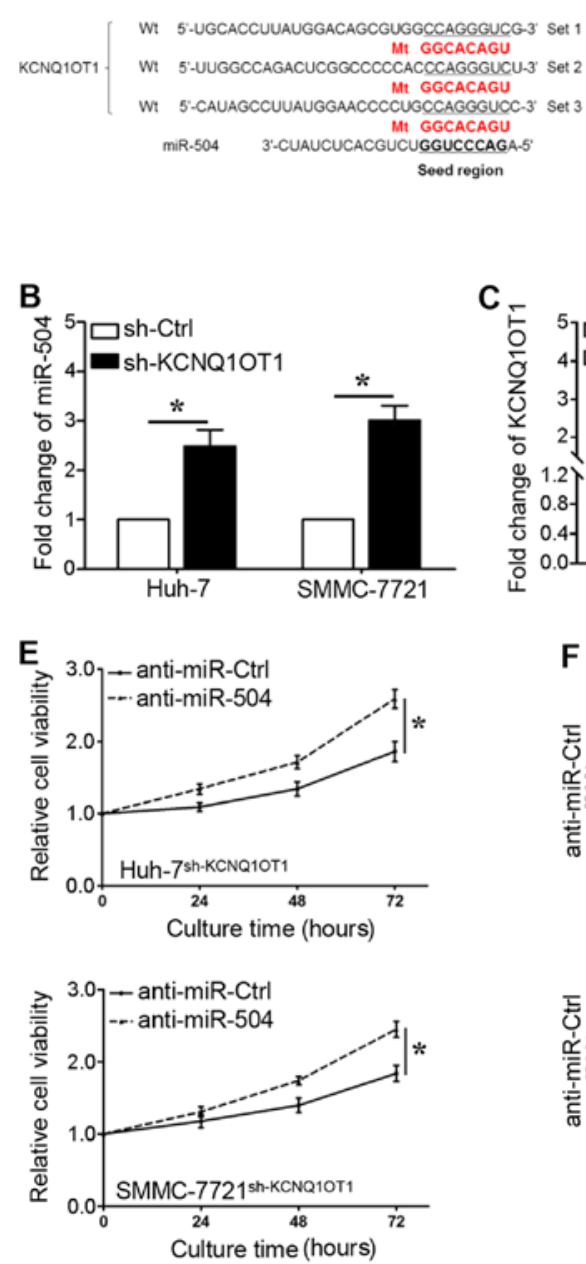
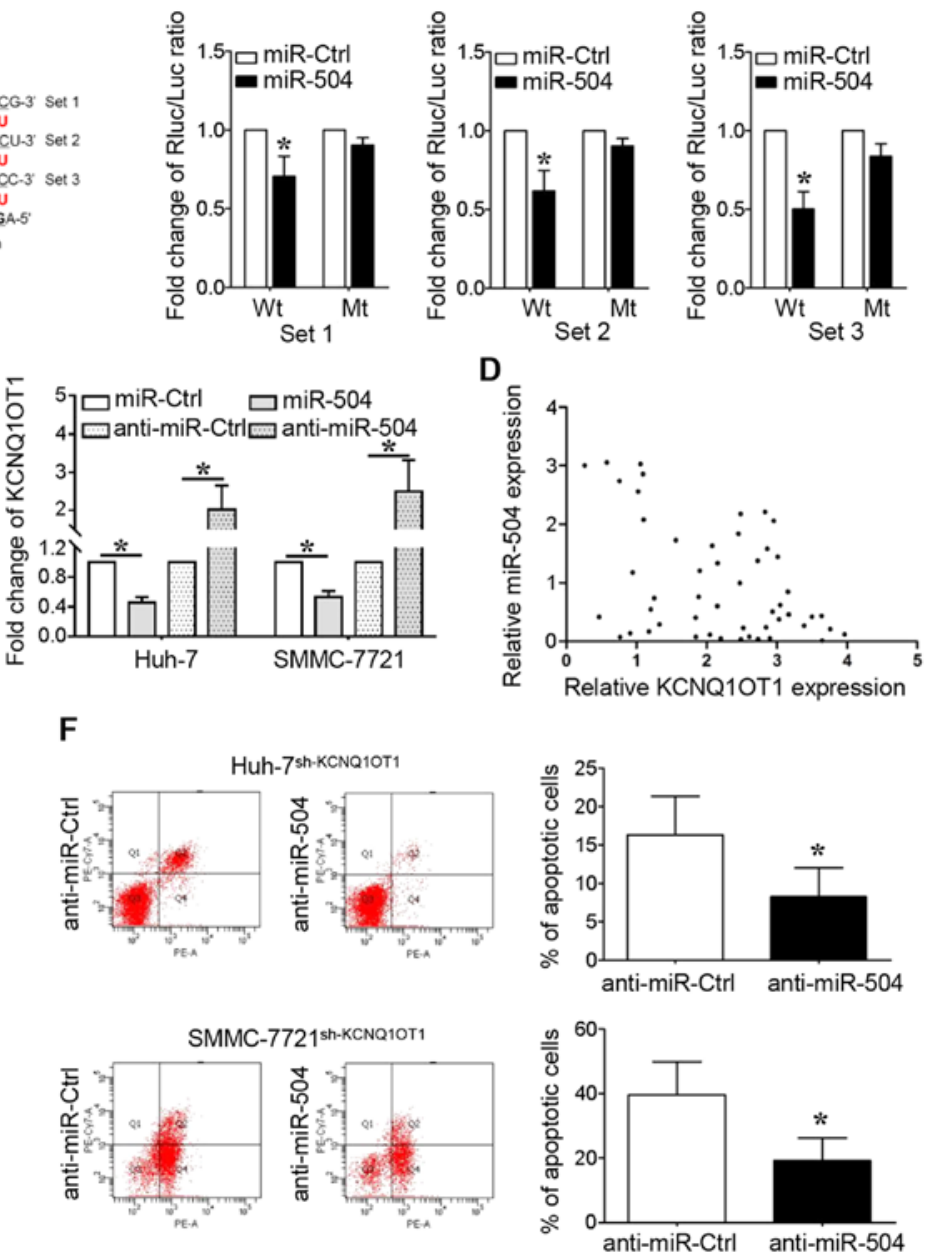

Figure 4. KCNQ1OT1 modulates cell proliferation by sponging miR-504. (A) miR-504 and its predicted binding sites in the KCNQ1OT1 sequence. Luciferase activities were detected to measure the binding of miR-504 to putative sites on KCNQ1OT1 following miR-504 mimic transfection. (B) The expression of miR-504 in Huh-7 and SMMC-7721 cells was determined following transfection with KCNQ1OT1 shRNA. (C) KCNQ1OT1 expression was detected in the Huh-7 and SMMC-7721 cells transfected with miR-504 mimics or miR-504 inhibitor. (D) Correlation between KCNQ1OT1 and miR-504 expression in HCC tissues. (E and F) Downregulation of miR-504 modulated (E) the viability and (F) the apoptosis of Huh-7 and SMMC-7721 cells transfected with KCNQ1OT1 shRNA. ${ }^{*} \mathrm{P}<0.05$. 
A

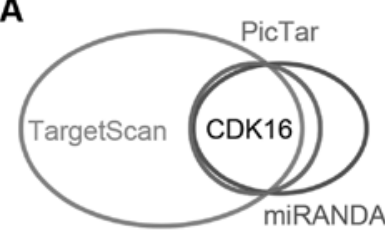

B

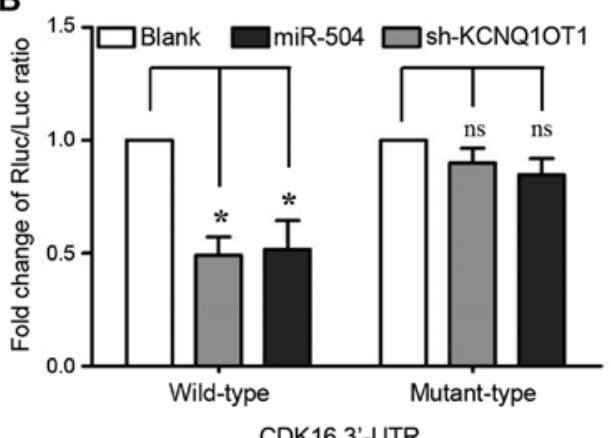

D
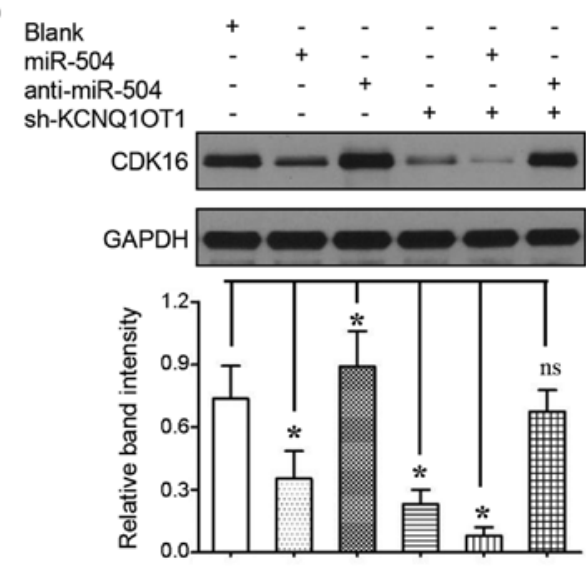

miR-504

3'-CUAUCUCACGUCUG'GUCCCAGA-5' IIIIIII CDK16 3'-UTR Wt 5'-GCCUCCUUCCUUCU'CAGGGUCU'-3' CDK16 3'-UTR Mt 5'-GCCUCCUUCCUUCU'AGUUCCAU'-3'

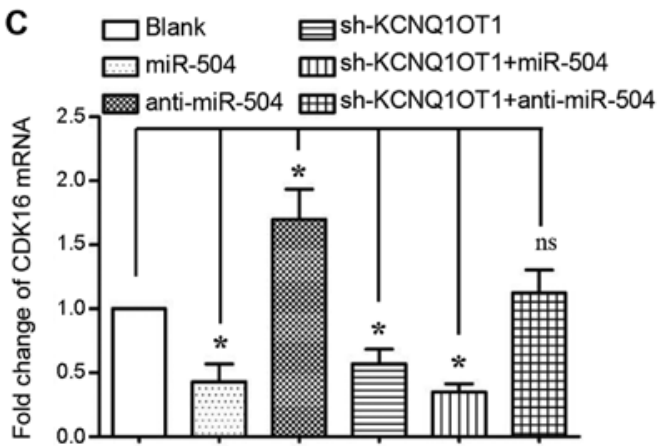

$\mathrm{E}$

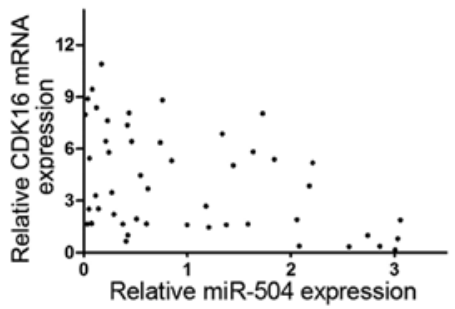

$\mathbf{F}$

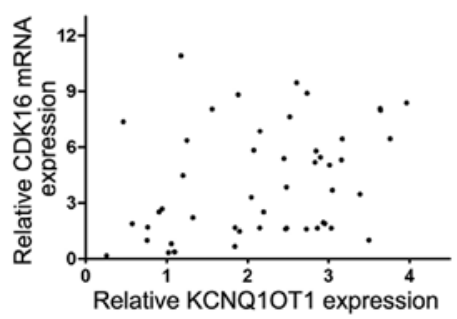

Figure 5. CDK16 is a direct target of the KCNQ1OT1/miR-504 axis. (A) CDK16 is a putative target of miR-504, as predicted by different algorithms. (B) Luciferase activities were determined to detect the binding of miR-504 to the putative sites in the 3'-UTR region of CDK16. (C and D) The expression of CDK16 (C) mRNA and (D) protein was measured following transfection with miR-504 mimics, miR-504 inhibitor, or KCNQ1OT1 shRNA, and co-transfection of miR-504 mimics with KCNQ1OT1 shRNA, or with the miR-504 inhibitor with KCNQ1OT1 shRNA. (E) Correlation between miR-504 and CDK16 mRNA expression in HCC tissues. (F) Correlation between KCNQ1OT1 and CDK16 mRNA expression in HCC tissues. ${ }^{*} \mathrm{P}<0.05$.

significant inverse correlation was verified by Spearman's correlation analysis between the miR-504 levels and the mRNA expression levels of CDK16 (Fig. 5E, r=-0.406; 95\% confidence interval, -0.6201 to $-0.1354 ; \mathrm{P}=0.003)$. Importantly, we also found that KNCQ1OT1 expression positively correlated with CDK16 mRNA expression (Fig. 5F, r=0.352; 95\% confidence interval, 0.073 to $0.580 ; \mathrm{P}=0.012)$. These results strongly indicate that CDK16 is a downstream target of miR-504 mediated by KCNQ1OT1 in HCC.

KCNQ1OT1 promotes GSK3 $\beta$ phosphorylation to decrease $\beta$-catenin-mediated Bcl-2 expression. To explore the underlying mechanisms of KCNQ1OT1/miR-504/CDK16, the GSK3 $\beta$ - $\beta$-catenin signaling pathway, which plays an important role in $\mathrm{HCC}$ progression, particularly in proliferation disorders (13), was evaluated in this study. KCNQ1OT1 knockdown decreased GSK3 $\beta$ phosphorylation and $\beta$-catenin expression in the Huh-7 (Fig. 6A, rows 1 and 3; $\mathrm{P}<0.05$ ) and SMMC-7721 (Fig. 6B, rows 1 and 3; $\mathrm{P}<0.05$ ) cells. We found that the expression of $\mathrm{Bcl}-2$ was also inhibited when KCNQ1OT1 was silenced in the Huh-7 (Fig. 6A, row 4,
$\mathrm{P}<0.05$ ) and SMMC-7721 (Fig. 6B, row 4, $\mathrm{P}<0.05$ ) cells. Importantly, the downregulation of miR-504 increased GSK3 $\beta$ phosphorylation, $\beta$-catenin expression and $\mathrm{Bcl}-2$ expression in the KCNQ1OT1 shRNA-transduced Huh-7 (Fig. 6C, P<0.05) and SMMC-7721 (Fig. 6D, P<0.05) cells. These data thus suggest that KCNQ1OT1 mediates HCC proliferation via the miR-504/CDK16/GSK3 $\beta$ signaling pathway.

\section{Discussion}

lncRNAs have critical biological functions, and KCNQ1OT1 is one of the few well-characterized lncRNAs. In the current study, we investigated the expression, clinical significance and biological functions of KCNQ1OT1 in human HCC. Several new discoveries were made. First, KCNQ1OT1 was found to be upregulated in human HCC tissues. This was consistent with findings that KCNQ1OT1 was overexpressed in a HCC cohort from the GSE dataset. Second, high levels KCNQ1OT1 were found to correlate with liver cirrhosis, an advanced TNM stage and a large tumor size. Tissues from larger-sized tumors ( $>5 \mathrm{~cm}$ in diameter) also had high 

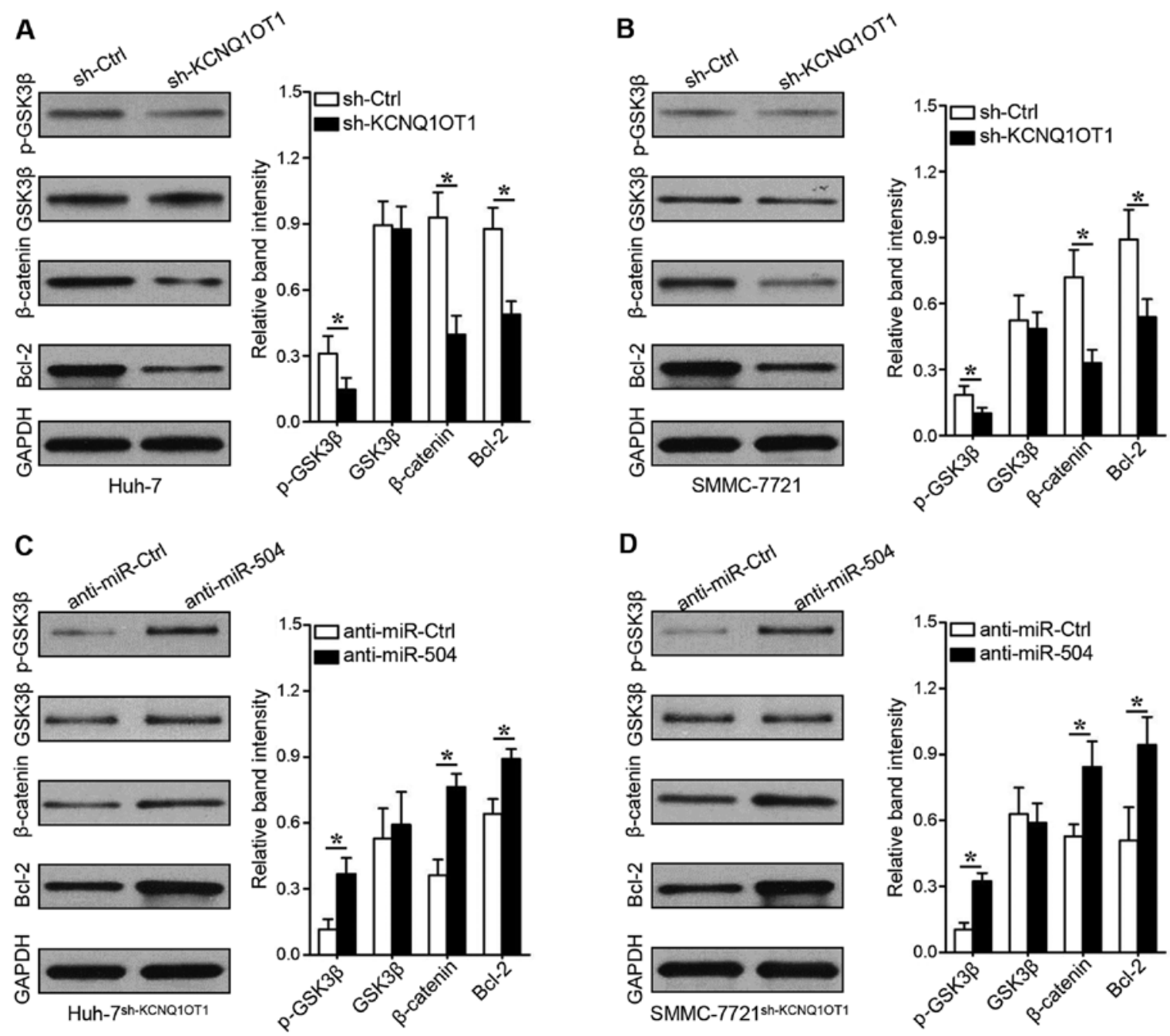

Figure 6. KCNQ1OT1 knockdown suppresses Bcl-2 expression by decreasing GSK3 $\beta$ phosphorylation at Ser9. (A and B) Western blot analysis of the expression of phosphorylated GSK3 $\beta$ at Ser9. Total GSK3 $\beta$, $\beta$-catenin and Bcl-2 following transfection with KCNQ1OT1 shRNA in (A) Huh-7 and (B) SMMC-7721 cells. (C and D) Western blot analysis of the expression of phosphorylated GSK3 $\beta$ at Ser9, total GSK3 $\beta$, $\beta$-catenin and Bcl-2 following co-transfection with KCNQ1OT1 shRNA and miR-504 inhibitor in (C) Huh-7 and (D) SMMC-7721 cells. "P<0.05.

KCNQ1OT1 expression levels. Third, patients with a high intra-tumoral KCNQ1OT1 expression experienced worse 3 -year overall survival and tumor-free survival times. This evidence strongly suggests that KCNQ1OT1 is a prognostic biomarker, and that it functions to promote tumor growth. With this antecedent, we subsequently found that the downregulation of KCNQ1OT1 inhibited cell proliferation and induced apoptosis in vitro, and attenuated subcutaneous tumor growth in vivo.

Consistent with our findings, Ren et al identified a high KCNQ1OT1 expression in lung adenocarcinoma tissues (14). KCNQ1OT1 knockdown suppressed the proliferation of and sensitized A549 cells to paclitaxel treatment. KCNQ1OT1 silencing also exerted tumor suppressive functions in glioma cells by acting as a sponge for miR-370 (15). The ability to function as a competing endogenous RNA (ceRNA) in order to sponge miRNAs is commonly found in lncRNA networks (16). For example, lncRNA-CCAT1 has been reported to sponge let-7 in HCC (17), miR-410 in glioma (18) and miR-181a in nasopharyngeal cancer (19). In this study, we found that KCNQ1OT1 had three fragments containing miR-504 seed region sequences. Upregulated miR-504 was found in cells in which KCNQ1OT1 was knocked down. The inhibition of miR-504 abrogated the changes in cell proliferation and apoptosis induced by KCNQ1OT1 knockdown. In the 50 pairs of HCC tissues, an inverse correlation was also confirmed between KCNQ1OT1 and miR-504 expression. We then used different algorithms to assume that CDK16 is a putative target of miR-504. The in vitro results from the dual-luciferase reporter gene system, RT-qPCR and western blot analyses verified our hypothesis. Moreover, the downregulation of KCNQ1OT1 inhibited CDK16 expression in HCC cells. Clinical analysis also confirmed the inverse correlation between KCNQ1OT1 and CDK16. Clearly, KCNQ1OT1 modulates CDK16 expression by sponging miR-504 in order to regulate cell proliferation in human HCC.

The underlying mechanisms through which KCNQ1OT1 increases HCC proliferation were also explored. CDKs are a family of serine/threonine-protein kinases. CDK16 is a recently recognized, atypical member of the CDK family (20). The activation of CDK16 usually occurs upon binding to membrane-associated cyclin Y (CCNY) (21). CDK16 has been reported to exert its functions by phosphorylating Akt at Ser473 (22), KAP0 at Ser83 (23) and caspase-8 at Ser387 (24). 
In this study, we found that KCNQ1OT1 silencing downregulated the phosphorylation of GSK3 $\beta$ at Ser9. Ser9 functions as an inhibitory phosphorylating position (25), which indicates that GSK3 $\beta$ phosphorylation was decreased by KCNQ1OT1 knockdown. Therefore, we detected the downregulation of $\beta$-catenin in the cells in which KCNQ1OT1 was knocked down. The expression of Bcl-2, an anti-apoptotic gene that can be regulated by $\beta$-catenin $(26,27)$, was also suppressed by transfection with KCNQ1OT1 shRNA. Notably, all these changes were attenuated by miR-504 inhibition. Our results suggested that the role of KCNQ1OT1 in the regulation of the GSK3 $\beta / \beta$-catenin/Bcl-2 signaling pathway depends on the inhibition of miR-504. Recently, Sunamura et al reported that $\beta$-catenin promoted KCNQ1OT1 transcription by directly binding to the KCNQ1OT1 promoter (28), which may explain why miR-504 overexpression decreased KCNQ1OT1 expression in Huh-7 and SMMC-7721 cells.

In conclusion, our data highlight the critical role played by KCNQ1OT1 in the proliferation and apoptosis of HCC cells. Our findings also indicate that lncRNA KCNQ1OT1 is a potential biomarker for HCC post-surgical surveillance, in addition to a molecular therapeutic target.

\section{Acknowledgements}

Not applicable.

\section{Funding}

This study was supported by a grant from the National Natural Scientific Foundation of China (no. 81472247).

\section{Availability of data and materials}

The analyzed datasets generated during the study are available from the corresponding author on reasonable request.

\section{Authors' contributions}

CLi designed the research and wrote the manuscript; RM, JZ and KQ participated in the research work; and CLiu designed the research.

\section{Ethics approval and consent to participate}

The use of clinical samples was approved by the Ethics Committee of the First Affiliated Hospital of Xi'an Jiaotong University. Written informed consent was obtained from all patients enrolled in this study. The in vivo experiments were performed after obtaining ethics approval from the Biomedical Ethics Committee of Xi'an Jiaotong University Health Science Center.

\section{Consent for publication}

Not applicable.

\section{Competing interests}

The authors declare that they have no competing interests.

\section{References}

1. Sim HW, Knox J and Dawson LA: An update on randomized clinical trials in hepatocellular carcinoma. Surg Oncol Clin N Am 26: 647-666, 2017.

2. Chen W, Zheng R, Baade PD, Zhang S, Zeng H, Bray F, Jemal A, Yu XQ and He J: Cancer statistics in China, 2015. CA Cancer J Clin 66: 115-132, 2016.

3. Kalogeraki A,Papadakis GZ, Tamiolakis D, Karvela-Kalogeraki I, Karvelas-Kalogerakis M, Segredakis J and Moustou E: Fine Needle Aspiration Biopsy (FNAB) in the Diagnosis of Hepatocellular Carcinoma: A Review. Rom J Intern Med 53: 209-217, 2015.

4. Chi HC, Tsai CY, Tsai MM, Yeh CT and Lin KH: Roles of long noncoding RNAs in recurrence and metastasis of radiotherapyresistant cancer stem cells. Int J Mol Sci 18: 18, 2017.

5. Klingenberg M, Matsuda A,Diederichs S and Patel T: Non-coding RNA in hepatocellular carcinoma: Mechanisms, biomarkers and therapeutic targets. J Hepatol 67: 603-618, 2017.

6. Huang MD, Chen WM, Qi FZ, Sun M, Xu TP, Ma P and Shu YQ: Long non-coding RNA TUG1 is up-regulated in hepatocellular carcinoma and promotes cell growth and apoptosis by epigenetically silencing of KLF2. Mol Cancer 14: 165, 2015.

7. Wei S, Fan Q, Yang L, Zhang X, Ma Y, Zong Z, Hua X, Su D, Sun H, Li H, et al: Promotion of glycolysis by HOTAIR through GLUT1 upregulation via mTOR signaling. Oncol Rep 38: 1902-1908, 2017.

8. Inoue T, Nakamura A, Matsubara K, Nyuzuki H, Nagasaki K, Oka A, Fukami M and Kagami M: Continuous hypomethylation of the KCNQ1OT1:TSS-DMR in monochorionic twins discordant for Beckwith-Wiedemann syndrome. Am J Med Genet A 173: 2847-2850, 2017.

9. Zhang Z, Weaver DL, Olsen D, deKay J, Peng Z, Ashikaga T and Evans MF: Long non-coding RNA chromogenic in situ hybridisation signal pattern correlation with breast tumour pathology. J Clin Pathol 69: 76-81, 2016.

10. Yoshizawa S, Fujiwara K, Sugito K, Uekusa S, Kawashima H, Hoshi R, Watanabe Y, Hirano T, Furuya T, Masuko T, et al: Pyrrole-imidazole polyamide-mediated silencing of KCNQ1OT1 expression induces cell death in Wilms' tumor cells. Int $\mathbf{J}$ Oncol 47: 115-121, 2015.

11. Livak KJ and Schmittgen TD: Analysis of relative gene expression data using real-time quantitative PCR and the 2(-Delta Delta C(T)) method. Methods 25: 402-408, 2001

12. Li C, Miao R, Liu S, Wan Y, Zhang S, Deng Y, Bi J, Qu K, Zhang J and Liu C: Down-regulation of miR-146b-5p by long noncoding RNA MALAT1 in hepatocellular carcinoma promotes cancer growth and metastasis. Oncotarget 8: 28683-28695, 2017.

13. Liu L, Zhang S, Hu L, Liu L, Guo W and Zhang J: HMGA1 participates in MHCC $97 \mathrm{H}$ cell proliferation and invasion through the ILK/Akt/GSK3 $\beta$ signaling pathway. Mol Med Rep 16: 9287-9294, 2017.

14. Ren K, Xu R, Huang J, Zhao J and Shi W: Knockdown of long non-coding RNA KCNQ1OT1 depressed chemoresistance to paclitaxel in lung adenocarcinoma. Cancer Chemother Pharmacol 80: 243-250, 2017.

15. Gong W, Zheng J, Liu X, Liu Y, Guo J, Gao Y, Tao W, Chen J, Li Z, Ma J, et al: Knockdown of long non-coding RNA KCNQ1OT1 restrained glioma cells' malignancy by activating miR-370/CCNE2 axis. Front Cell Neurosci 11: 84, 2017.

16. Qu J, Li M, Zhong W and Hu C: Competing endogenous RNA in cancer: A new pattern of gene expression regulation. Int J Clin Exp Med 8: 17110-17116, 2015.

17. Deng L, Yang SB, Xu FF and Zhang JH: Long noncoding RNA CCAT1 promotes hepatocellular carcinoma progression by functioning as let-7 sponge. J Exp Clin Cancer Res 34: 18, 2015.

18. Wang ZH, Guo XQ, Zhang QS, Zhang JL, Duan YL, Li GF and Zheng DL: Long non-coding RNA CCAT1 promotes glioma cell proliferation via inhibiting microRNA-410. Biochem Biophys Res Commun 480: 715-720, 2016.

19. Wang Q, Zhang W and Hao S: LncRNA CCAT1 modulates the sensitivity of paclitaxel in nasopharynx cancers cells via miR-181a/CPEB2 axis. Cell Cycle 16: 795-801, 2017.

20. Mikolcevic P, Rainer J and Geley S: Orphan kinases turn eccentric: A new class of cyclin Y-activated, membrane-targeted CDKs. Cell Cycle 11: 3758-3768, 2012.

21. Shehata SN, Deak M, Morrice NA, Ohta E, Hunter RW, Kalscheuer VM and Sakamoto K: Cyclin Y phosphorylation- and 14-3-3-binding-dependent activation of PCTAIRE-1/CDK16. Biochem J 469: 409-420, 2015. 
22. Ćwiek P, Leni Z, Salm F, Dimitrova V, Styp-Rekowska B, Chiriano G, Carroll M, Höland K, Djonov V, Scapozza L, et al: RNA interference screening identifies a novel role for PCTK1/CDK16 in medulloblastoma with c-Myc amplification. Oncotarget 6: 116-129, 2015.

23. Iwano S, Satou A, Matsumura S, Sugiyama N, Ishihama Y and Toyoshima F: PCTK1 regulates integrin-dependent spindle orientation via protein kinase A regulatory subunit KAP0 and myosin X. Mol Cell Biol 35: 1197-1208, 2015.

24. Yanagi T, Shi R, Aza-Blanc P, Reed JC and Matsuzawa S: PCTAIRE1-knockdown sensitizes cancer cells to TNF family cytokines. PLoS One 10: e0119404, 2015.

25 . Huang L, Zhang C, Su L and Song Z: GSK3 $\beta$ attenuates TGF- $\beta 1$ induced epithelial-mesenchymal transition and metabolic alterations in ARPE-19 cells. Biochem Biophys Res Commun 486 744-751, 2017.
26. Ye X, Lin J, Lin Z, Xue A, Li L, Zhao Z, Liu L, Shen Y and Cong B: Axin1 up-regulated 1 accelerates stress-induced cardiomyocytes apoptosis through activating $\mathrm{Wnt} / \beta$-catenin signaling. Exp Cell Res 359: 441-448, 2017.

27. Wang Y, Zhou J, Xu YJ and Hu HB: Long non-coding RNA LINC00968 acts as oncogene in NSCLC by activating the Wnt signaling pathway. J Cell Physiol 233: 3397-3406, 2018.

28. Sunamura N, Ohira T, Kataoka M, Inaoka D, Tanabe H, Nakayama Y, Oshimura M and Kugoh H: Regulation of functional KCNQ1OT1 lncRNA by $\beta$-catenin. Sci Rep 6: 20690, 2016. 